\title{
Oscillations in Plankton Models with Nutrient Recycling
}

\author{
SHIGUi RUAN* $\dagger$ \\ Department of Mathematics and Statistics and School of Biomedical Engineering, Dalhousie University, \\ Halifax, Nova Scotia, Canada B3H $3 J 5$
}

(Received on 25 January 1999, Accepted in revised form on 6 September 2000)

\begin{abstract}
Plankton-nutrient interaction models with both instantaneous and delayed nutrient recycling are considered. The system consists of three components: autotrophic phytoplankton, herbivorous zooplankton and dissolved limiting nutrient. Local stability of the equilibria is analysed. It is shown that the positive equilibrium loses its stability when the nutrient input concentration passes through a critical value and the Hopf bifurcation occurs that induces oscillations of the populations. Numerical simulations are carried out to illustrate the obtained results.
\end{abstract}

(C) 2001 Academic Press

\section{Introduction}

An important difference between a chemostat situation and a lake situation is that lakes generally have a residence time of nutrient and sediments measured in years (see Powell \& Richerson, 1985). This means that in models of natural systems the regeneration of nutrient due to bacterial decomposition of the dead biomass must be considered (see Svirezhev and Logofet, 1983).

The effect of nutrient recycling on ecosystem stability has been extensively studied for closed systems. Usually, nutrient recycling is considered as an instantaneous term, thus the time required to regenerate nutrient from dead biomass by bacterial decomposition is neglected. In a natural system, such a delay is always present and increases as temperature decreases (Whittaker, 1975).

Beretta et al. (1990) considered an open system with a single species feeding on a limiting nutrient which is partially recycled after the death of the organisms. They inserted a distributed delay in

\footnotetext{
* E-mail: ruan@mscs.dal.ca

$\uparrow$ Research was supported by the NSERC of Canada and the Petro-Canada Young Innovator Award.
}

the recycling term in order to study its effect on the stability of the positive equilibrium. Bischi (1992) studied the effects of the time delay involved in nutrient recycling on resilience, that is, the rate at which a system returns to a stable steady state following a perturbation. Bischi showed that when a system is characterized by oscillatory behavior, an increase in the distributed time delay can have a stabilizing effect. This is a counterintuitive result, because in general it has been found that the introduction of time delays is a destabilizing process, in the sense that increasing the time delay could cause a stable equilibrium to become unstable and/or cause the populations to fluctuate (see Cushing, 1977). Recently, Beretta \& Takeuchi (1994a, b) and He \& Ruan (1998) studied the global stability of this model by applying the Liapunov functional method. For other related work, we refer to $\mathrm{He}$ et al. (1998), Ruan (1995) and the references cited therein. Freedman \& Xu (1993) extended the single species model in Beretta et al. (1990) to a competition model of chemostat-type with delayed nutrient recycling. They developed persistence and extinction criteria for the competing 
populations. The global stability of the chemostat-type competition models with nutrient recycling has been investigated in Ruan and He (1998).

In the last two decades, numerous plankton models with instantaneous nutrient recycling have been proposed (see Busenberg et al., 1990; DeAngelis et al., 1989; Evan \& Parslow, 1985; Fasham, 1993; Fasham et al., 1990; Franks et al., 1986; Henderson \& Steele, 1995; Nisbet \& Gurney, 1976; Nisbet et al., 1983; Ruan, 1993; Steele \& Henderson, 1981, 1992; Sarmiento et al., 1993; Truscott \& Brindley, 1994; Wroblewski et al. 1988). In 1995, Beretta et al. and Ruan \& Wolkowicz (1995) simultaneously proposed a plankton-nutrient model with delayed nutrient recycling. The system is consisted of autotrophic phyto-plankton, herbivorous zooplankton and dissolved limiting nutrient. Distributed delays are used to describe the contribution of phytoplankton and zooplankton that died in the past to the nutrient recycled at the present time. While Beretta et al. (1995) were interested in boundedness and local stability of the system, Ruan \& Wolkowicz (1995) gave uniform persistence criteria for the system. In this paper, we first consider the model with instantaneous nutrient recycling and analyse the local stability of the equilibria. By choosing $N^{0}$, the nutrient input concentration, as a bifurcation parameter, it is shown that the positive equilibrium loses its stability when $N^{0}$ passes through a critical value and a family of periodic solutions bifurcates from the positive equilibrium via the Hopf bifurcation. Then, we consider the model with delayed nutrient recycling and observe similar oscillations in the plankton populations. Numerical simulations are given to illustrate the results and observations.

\section{The Model}

The plankton model with delayed nutrient recycling consists of three interacting components, herbivorous zooplankton $(Z)$, autotrophic phytoplankton $(P)$ and dissolved limiting nutrient $(N)$, and is given by the following equations:

$$
\begin{aligned}
\frac{\mathrm{d} N}{\mathrm{~d} t}= & D\left(N^{0}-N\right)-a P u(N) \\
& +(1-\delta) c Z w(P)
\end{aligned}
$$

$$
\begin{gathered}
+\gamma_{1} \int_{-\infty}^{t} F(t-s) P(s) \mathrm{d} s \\
+\varepsilon_{1} \int_{-\infty}^{t} G(t-s) Z(s) \mathrm{d} s, \\
\frac{\mathrm{d} P}{\mathrm{~d} t}=a P u(N)-c Z w(P)-\left(\gamma+D_{1}\right) P, \\
\frac{\mathrm{d} Z}{\mathrm{~d} t}=Z\left[\delta c w(P)-\left(\varepsilon+D_{2}\right)\right],
\end{gathered}
$$

where all parameters are positive and are interpreted as follows:

$a$ - maximal nutrient uptake rate for the phytoplankton

$c$-maximal zooplankton ingestion rate

$N^{0}$-input concentration of the nutrient

$D$-washout rate of the system

$D_{1}$ - washout rate of the phytoplankton

$\mathrm{D}_{2}$ - washout rate of the zooplankton

$\gamma$-phytoplankton mortality rate

$\varepsilon$-zooplankton death rate

$\gamma_{1}$-nutrient recycle rate after the death of the phytoplankton, $\gamma_{1} \leqslant \gamma$

$\varepsilon_{1}$ - nutrient recycle rate after the death of the zooplankton, $\varepsilon_{1} \leqslant \varepsilon$

$\delta$ - the fraction of zooplankton nutrient conversion, $0<\delta \leqslant 1$.

The function $u(N)$ describes the nutrient uptake rate of phytoplankton and satisfies the following general hypotheses (Hale \& Somolinos, 1983):

(i) the function is nonnegative, increasing and vanishes when there is no nutrient;

(ii) there is a saturation effect when the nutrient is very abundant.

That is, $u(N)$ is a continuous function defined on $[0, \infty)$ and satisfies

$$
u(0)=0, \quad \frac{\mathrm{d} u}{\mathrm{~d} N}>0 \quad \text { and } \quad \lim _{N \rightarrow \infty} u(N)=1 .
$$

In particular, this kind of function includes Holling's type II (Holling, 1959) or the MichaelisMenten function (Wroblewski \& Richman, 1987)

$$
u(N)=\frac{N}{k+N},
$$


where $k$ is the half-saturation constant or Michaelis-Menten constant.

The function $w(P)$ represents the response function describing herbivore grazing. It is also assumed that $w(P)$ is continuous on $[0, \infty)$ and satisfies

$$
w(0)=0, \quad \frac{\mathrm{d} w}{\mathrm{~d} P}>0 .
$$

Usually, Ivlev's (1961) functional response formulation

$$
w(P)=1-\mathrm{e}^{-\lambda P}
$$

is used to describe the zooplankton grazing, where $\lambda$ is the rate at which saturation is achieved with increasing phytoplankton levels (per unit concentration). Alternatively, Mayzaud and Poulet formulation (Mayzaud \& Poulet, 1978)

$$
w(P)=\lambda P\left(1-\mathrm{e}^{-\lambda P}\right)
$$

is also used to describe the food-acclimatized herbivore grazing (Wroblewski \& Richman, 1987).

The delay-kernels $F(s)$ and $G(s)$ are nonnegative bounded functions defined on $[0, \infty)$ and describe the contribution of phytoplankton and zooplankton that died in the past to the nutrient recycled at time $t$. We assume that only fractions of the dead phytoplankton $\left(\gamma_{1}\right)$ and the dead zooplankton $\left(\varepsilon_{1}\right)$ are recycled into dissolved nutrient. The presence of the distributed time delay must not affect the equilibrium values, so we normalize the kernels such that

$$
\int_{0}^{\infty} F(s) \mathrm{d} s=1, \quad \int_{0}^{\infty} G(s) \mathrm{d} s=1 .
$$

According to MacDonald (1978), we define the average time lag as

$$
T=\int_{0}^{\infty} s F(s) \mathrm{d} s .
$$

In particular, the exponential kernel

$$
\frac{\alpha^{n+1}}{n !} s^{n} \mathrm{e}^{-\alpha s}, \quad \alpha>0
$$

is usually used (see Cushing, 1977), where $n$ is a nonnegative integer and $\alpha$ is linked to the mean time delay by

$$
T=\frac{n+1}{\alpha} .
$$

During consumption, only a fraction of the biomass removed from the resources compartment, $\delta(\delta \leqslant 1)$, is assumed to be assimilated by the consumer. The remainder goes directly to the dissolved nutrient. Besides the loss related to consumption, a second phytoplankton loss term, $-\gamma P$, represents loss due to extracellular release and senescent cell autolysis and sinking. The zooplankton dynamics includes growth as assimilated ingested ration and a loss rate $(\varepsilon)$ due to high-level predation, physiological death, etc. The parameters $D, D_{1}, D_{2}$ are the washout rates (or removal rates, diffusive rates) of biotic components from the system resulting from washout, diffusion, harvesting, burial in deep sediments, soluble metabolic loss or cell sinking, for example. Those processes in general do not take place for the same amount of time, so we assume that $D, D_{1}, D_{2}$ are different.

System (1) with initial conditions

$$
\begin{gathered}
N(0)=N_{0} \geqslant 0, \quad P(s)=\psi(s), \quad Z(s)=\psi(s), \\
-\infty<s \leqslant 0,
\end{gathered}
$$

where $\phi, \psi:(-\infty, 0] \rightarrow[0, \infty)$ are bounded and continuous functions, possesses unique nonnegative solutions continuously dependent on parameters and initial data (see Cushing, 1977).

\section{Instantaneous Nutrient Recycling}

We first assume that $F(s)=G(s)=\delta(s)$, the Dirac delta function, that is, we neglect the time delays involved in the nutrient recycling process. System (1) becomes a set of three ordinary differential equations (Ruan, 1993)

$$
\begin{aligned}
\frac{\mathrm{d} N}{d t}= & D\left(N^{0}-N\right)-a P u(N)+(1-\delta) c Z w(P) \\
& +\gamma_{1} P+\varepsilon_{1} Z
\end{aligned}
$$




$$
\begin{aligned}
& \frac{\mathrm{d} P}{\mathrm{~d} t}=a P u(N)-c Z w(P)-\left(\gamma+D_{1}\right) P, \\
& \frac{\mathrm{d} Z}{\mathrm{~d} t}=Z\left[\delta c w(P)-\left(\varepsilon+D_{2}\right)\right]
\end{aligned}
$$

and the initial conditions become

$N(0)=N_{0} \geqslant 0, \quad P(0)=P_{0} \geqslant 0, \quad Z(0)=Z_{0} \geqslant 0$.

Adding all the three equations, we can see that

$$
\begin{aligned}
\frac{\mathrm{d}}{\mathrm{d} t}(N+P+Z)= & D N^{0}-D N+\gamma_{1} P+\varepsilon_{1} Z \\
& -\gamma P-D_{1} P-\varepsilon Z-D_{2} Z \\
\leqslant & -D_{0}\left((N+P+Z)+D N^{0}\right),
\end{aligned}
$$

where $D_{0}=\min \left\{D, D_{1}, D_{2}\right\}$. It follows that the solutions are bounded.

Proposition 3.1. All solutions of system (7) under (8) are bounded.

Notice that the boundary equilibrium $E_{0}=\left(N^{0}, 0,0\right)$ always exists. To determine the local stability of $E_{0}$, we consider the Jacobian matrix of the linearized system at $E_{0}$,

$$
M_{0}=\left(\begin{array}{ccc}
-D & -a u\left(N^{0}\right)+\gamma_{1} & \varepsilon_{1} \\
0 & a u\left(N^{0}\right)-\left(\gamma+D_{1}\right) & 0 \\
0 & 0 & -\left(\varepsilon+D_{2}\right)
\end{array}\right)
$$

The eigenvalues of $M_{0}$ are

$$
\begin{gathered}
y_{1}=-D<0, \quad y_{2}=a u\left(N^{0}\right)-\left(\gamma+D_{1}\right), \\
y_{3}=-\left(\varepsilon+D_{2}\right)<0 .
\end{gathered}
$$

Hence, the local stability of $E_{0}$ can be determined.

\section{Proposition 3.2. If}

$$
a u\left(N^{0}\right)-\left(\gamma+D_{1}\right)<0,
$$

then $E_{0}=\left(N^{0}, 0,0\right)$ is asymptotically stable.

Note that inequality (9) is equivalent to

$$
a<\gamma+D_{1} \quad \text { and } \quad N^{0}<u^{-1}\left(\frac{\gamma+D_{1}}{a}\right) .
$$

Recall that $a$ is the maximal nutrient uptake rate of the phytoplankton and $N^{0}$ is the input concentration of the nutrient. Proposition 3.2 indicates that if the maximal nutrient uptake rate of the phytoplankton population is less then its loss rate $\left(\gamma+D_{1}\right)$ and there is not enough nutrient input into the system, than the phytoplankton population and therefore the zooplankton population cannot survive. Hence, to have the instability of $E_{0}$, we assume that

$$
a>\gamma+D_{1} \quad \text { and } \quad N^{0}>u^{-1}\left(\frac{\gamma+D_{1}}{a}\right) .
$$

It then follows that $y_{2}>0$ and $E_{0}$ is an unstable saddle, the $(N, Z)$-plane is the stable manifold and the $P$-axis is the unstable manifold.

The inequalities in eqn (10) also imply the existence of the boundary steady state $E_{1}=\left(N_{1}, P_{1}, 0\right)$, where

$$
N_{1}=u^{-1}\left(\frac{\gamma+D_{1}}{a}\right), \quad P_{1}=\frac{D\left(N^{0}-N_{1}\right)}{\gamma+D_{1}-\gamma_{1}} .
$$

If $E_{1}$ exists, then the second inequality in eqn (10) implies that

$$
N^{0}>N_{1} \text {. }
$$

The Jacobian matrix at $E_{1}$ is

$$
M_{1}=\left(\begin{array}{ccc}
-D-a P_{1} u^{\prime}\left(N_{1}\right) & -a u\left(N_{1}\right)+\gamma_{1} & (1-\delta) c w\left(P_{1}\right)+\varepsilon_{1} \\
a P_{1} u^{\prime}\left(N_{1}\right) & 0 & -c w\left(P_{1}\right) \\
0 & 0 & \delta c w\left(P_{1}\right)-\left(\varepsilon_{1}+D_{2}\right)
\end{array}\right)
$$


Let $y_{1}, y_{2}$ and $y_{3}$ be the three eigenvalues of the matrix $M_{1}$. Then we can see that

$$
\begin{aligned}
\operatorname{Re} y_{1,2} & =-\left(D+a P_{1} u^{\prime}\left(N_{1}\right)\right)<0, \\
y_{3} & =\delta c w\left(P_{1}\right)-\left(\varepsilon_{1}+D_{2}\right)
\end{aligned}
$$

and state the following result about the stability of $E_{1}$.

\section{Proposition 3.3. If}

$$
\delta c w\left(P_{1}\right)-\left(\varepsilon_{1}+D_{2}\right)<0,
$$

then $E_{1}=\left(N_{1}, P_{1}, 0\right)$ is asymptotically stable.

Taking the biological meanings of the parameters involved in the inequality (11) into consideration, Proposition 3.3 then implies that if the growth rate of the zooplankton population is less than its loss rate, then only the phytoplankton population can survive on the nutrient, the zooplankton will die out. Again, to have the instability of $E_{1}$, we assume that

$\delta c>\varepsilon_{1}+D_{2} \quad$ and $\quad P_{1}>w^{-1}\left(\frac{\varepsilon_{1}+D_{2}}{\delta c}\right)$.

Hence, $y_{3}>0$ and $E_{1}$ is also an unstable saddle, the $(N, P)$-plane is the stable manifold and the $\mathrm{Z}$-axis is the unstable manifold. Moreover, if the inequalities in eqn (9) are satisfied, then there exists an interior steady state $E^{*}=\left(N^{*}, P^{*}, Z^{*}\right)$ with

$$
P^{*}=w^{-1}\left(\frac{\varepsilon_{1}+D_{2}}{\delta c}\right)
$$

Notice that the second inequality in eqns (12) and (13) imply that

$$
P_{1}>P^{*}
$$

The Jacobian matrix at $E^{*}$ is given by

$$
M^{*}=\left(\begin{array}{ccc}
m_{11} & m_{12} & m_{13} \\
m_{21} & m_{22} & m_{23} \\
0 & m_{32} & 0
\end{array}\right),
$$

where

$$
\begin{aligned}
& m_{11}=-D-a P^{*} u^{\prime}\left(N^{*}\right)<0, \\
& m_{12}=-a u\left(N^{*}\right)+(1-\delta) c Z^{*} w^{\prime}\left(P^{*}\right)+\gamma_{1}, \\
& m_{13}=(1-\delta) c w\left(P^{*}\right)+\varepsilon_{1}>0, \\
& m_{21}=a P^{*} u^{\prime}\left(N^{*}\right)>0, \\
& m_{22}=a u\left(N^{*}\right)-c Z^{*} w^{\prime}\left(P^{*}\right)-\left(\gamma+D_{1}\right), \\
& m_{23}=-c w\left(P^{*}\right)<0, \\
& m_{32}=\delta c Z^{*} w^{\prime}\left(P^{*}\right)>0 .
\end{aligned}
$$

The characteristic equation is

$$
y^{3}+a_{1} y^{2}+a_{2} y+a_{3}=0,
$$

where

$a_{1}=-\left(m_{11}+m_{22}\right)$,

$a_{2}=m_{11} m_{22}-m_{12} m_{21}-m_{23} m_{32}$,

$a_{3}=m_{11} m_{23} m_{32}-m_{13} m_{32} m_{21}$.

By the Routh-Hurwitz criteria, all roots of eqn (15) have negative real parts if and only if

$$
a_{1}>0, \quad a_{3}>0 \text { and } a_{1} a_{2}-a_{3}>0 .
$$

Assume

$$
m_{22}<0
$$

then $a_{1}>0, a_{3}>0$ and

$$
\begin{aligned}
m_{12} & \leqslant-a u\left(N^{*}\right)+(1-\delta) c Z^{*} w^{\prime}\left(P^{*}\right)+\gamma \\
& =m_{22}-D-1-\delta c Z^{*} w^{\prime}\left(P^{*}\right)<0 .
\end{aligned}
$$

By the signs of the $m_{i j}$ 's, we also have

$$
\begin{aligned}
a_{1} a_{2}-a_{3}= & -m_{11}^{2} m_{22}-m_{11} m_{22}^{2}+m_{11} m_{12} m_{21} \\
& +m_{22} m_{12} m_{21}+m_{11} m_{23} m_{32} \\
& +m_{22} m_{23} m_{32} \\
> & 0
\end{aligned}
$$


since each term is positive. This yields the following result on stability of $E^{*}$.

Proposition 3.4. The positive steady state $E^{*}$ is asymptotically stable if

$$
a u\left(N^{*}\right)<c Z^{*} w^{\prime}\left(P^{*}\right)+\left(\gamma+D_{1}\right) .
$$

Now assume that the steady state $E^{*}$ is asymptotically stable, we would like to know if $E^{*}$ will lose its stability when one of the parameters changes. Note that the asymptotic stability of $E^{*}$ is equivalent to the conditions $a_{1}>0, a_{3}>0$ and $a_{1} a_{2}>a_{3}$. We want to know that when one of the parameters changes, if the conditions (more specifically if the condition $a_{1} a_{2}>a_{3}$ ) will be violated. Furthermore, we want to determine if the real part of the roots of the characteristic eqn (15) changes from negative to zero and to positive, that is, if the Hopf bifurcation will occur at $E^{*}$.

Choose $N^{0}$, the input of concentration, as the bifurcation parameter, we can see that if there exists a critical value $N_{c}^{0}$ such that

$$
\begin{gathered}
a_{1}\left(N_{c}^{0}\right)>0, \quad a_{1}\left(N_{c}^{0}\right) a_{2}\left(N_{c}^{0}\right)=a_{3}\left(N_{c}^{0}\right), \\
{\left[a_{1}\left(N_{c}^{0}\right) a_{2}\left(N_{c}^{0}\right)\right]^{\prime}<a_{3}^{\prime}\left(N_{c}^{0}\right),}
\end{gathered}
$$

then the characteristic equation (15) can be written as

$$
\left(y^{2}\left(N_{c}^{0}\right)+a_{2}\left(N_{c}^{0}\right)\right)\left(y\left(N_{c}^{0}\right)+a_{1}\left(N_{c}^{0}\right)\right)=0,
$$

which has roots

$$
y_{1,2}\left(N_{c}^{0}\right)= \pm i \sqrt{a_{2}\left(N_{c}^{0}\right)}, \quad y_{3}=-a_{1}\left(N_{c}^{0}\right)<0 .
$$

To see if Hopf bifurcation occurs at $N_{0}=N_{c}^{0}$, we need to verify the transversality condition

$$
\left.\frac{\mathrm{dRe} y_{1,2}}{\mathrm{~d} N^{0}}\right|_{N^{0}=N_{c}^{0}} \neq 0 .
$$

Assume that the pair of conjugate complex roots of eqn (15) has the form $y_{1,2}\left(N^{0}\right)=\mu\left(N^{0}\right) \pm$ iv $\left(N^{0}\right)$. Substituting into eqn (15) and calculating the derivatives, we obtain

$$
\begin{aligned}
& A\left(N^{0}\right) \mu^{\prime}\left(N^{0}\right)-B\left(N^{0}\right) v^{\prime}\left(N^{0}\right)+C\left(N^{0}\right)=0, \\
& B\left(N^{0}\right) \mu^{\prime}\left(N^{0}\right)+A\left(N^{0}\right) v^{\prime}\left(N^{0}\right)+D\left(N^{0}\right)=0,
\end{aligned}
$$

where

$$
\begin{aligned}
A\left(N^{0}\right)= & 3 \mu^{2}\left(N^{0}\right)+2 a_{1}\left(N^{0}\right) \mu\left(N^{0}\right) \\
& +a_{2}\left(N^{0}\right)-3 v^{2}\left(N^{0}\right), \\
B\left(N^{0}\right)= & 6 \mu\left(N^{0}\right) v\left(N^{0}\right)+2 a_{1}\left(N^{0}\right) v\left(N^{0}\right), \\
C\left(N^{0}\right)= & \mu^{2}\left(N^{0}\right) a_{1}^{\prime}\left(N^{0}\right)+a_{2}^{\prime}\left(N^{0}\right) \mu\left(N^{0}\right) \\
& +a_{3}^{\prime}\left(N^{0}\right)-a_{1}^{\prime}\left(N^{0}\right) v^{2}\left(N^{0}\right), \\
D\left(N^{0}\right)= & 2 \mu\left(N^{0}\right) v\left(N^{0}\right) a_{1}^{\prime}\left(N^{0}\right) \\
& +a_{2}^{\prime}\left(N^{0}\right) v\left(N^{0}\right) .
\end{aligned}
$$

Notice that $\mu\left(N_{c}^{0}\right)=0, v\left(N_{c}^{0}\right)=\sqrt{a_{2}\left(N_{c}^{0}\right)}$, we have

$$
A\left(N_{c}^{0}\right)=-2 a_{2}\left(N_{c}^{0}\right), \quad B\left(N_{c}^{0}\right)=2 a_{1}\left(N_{c}^{0}\right) \sqrt{a_{2}\left(N_{c}^{0}\right)},
$$

$C\left(N_{c}^{0}\right)=a_{3}^{\prime}\left(N_{c}^{0}\right)-a_{1}^{\prime}\left(N_{c}^{0}\right) a_{2}\left(N_{c}^{0}\right)$,

$D\left(N_{c}^{0}\right)=a_{2}^{\prime}\left(N_{c}^{0}\right) \sqrt{a_{2}\left(N_{c}^{0}\right) .}$

Solving for $\mu^{\prime}\left(N_{c}^{0}\right)$ from system (19), we obtain

$$
\begin{aligned}
& \left.\frac{\mathrm{dRe} y_{1,2}}{\mathrm{~d} N^{0}}\right|_{N^{0}=N_{c}^{0}}=\left.\mu^{\prime}\left(N^{0}\right)\right|_{N^{0}=N_{c}^{0}} \\
& =-\frac{A\left(N_{c}^{0}\right) C\left(N_{c}^{0}\right)+B\left(N_{c}^{0}\right) D\left(N_{c}^{0}\right)}{A\left(N_{c}^{0}\right)^{2}+B\left(N_{c}^{0}\right)^{2}} \\
& =\frac{a_{3}^{\prime}\left(N_{c}^{0}\right)-a_{1}^{\prime}\left(N_{c}^{0}\right) a_{2}\left(N_{c}^{0}\right)-a_{1}\left(N_{c}^{0}\right) a_{2}^{\prime}\left(N_{c}^{0}\right)}{a_{1}^{2}\left(N_{c}^{0}\right)+a_{2}\left(N_{c}^{0}\right)}
\end{aligned}
$$

$>0$. 
Thus, the real part of $y_{1,2}$ changes from negative to zero at $N^{0}=N_{c}^{0}$ and becomes positive when $N^{0}>N_{c}^{0}$. Summarizing the above analysis, we have the following result on the bifurcation of system (7).

Proposition 3.5. If there exists a positive number $N_{c}^{0}$ such that $a_{1}\left(N_{c}^{0}\right)>0, a_{1}\left(N_{c}^{0}\right) a_{2}\left(N_{c}^{0}\right)=a_{3}\left(N_{c}^{0}\right)$ and $\left[a_{1}\left(N_{c}^{0}\right) a_{2}\left(N_{c}^{0}\right)\right]^{\prime}<a_{3}^{\prime}\left(N_{c}^{0}\right)$, then when $N^{0}<$ $N_{c}^{0}$, the steady state $E^{*}$ is stable; when $N^{0}=N_{c}^{0}$, $E^{*}$ loses its stability and the Hopf bifurcation occurs at $E^{*}$; when $N^{0}>N_{c}^{0}, E^{*}$ becomes unstable and a family of periodic solutions bifurcates from $E^{*}$.

As an example, we suppose that $u(N)$ is the Michaelis-Menten function, $w(P)$ is the Ivlev function, i.e. we consider the following model:

$$
\begin{aligned}
\frac{\mathrm{d} N}{\mathrm{~d} t}= & D\left(N^{0}-N\right)-a P \frac{N}{k+N} \\
& +(1-\delta) c Z\left(1-\mathrm{e}^{-\lambda P}\right)+\gamma_{1} P+\varepsilon_{1} Z,
\end{aligned}
$$$$
\frac{\mathrm{d} P}{\mathrm{~d} t}=a P \frac{N}{k+N}-c Z\left(1-\mathrm{e}^{-\lambda P}\right)-\left(\gamma+D_{1}\right) P,
$$$$
\frac{\mathrm{d} Z}{\mathrm{~d} t}=Z\left[\delta c\left(1-\mathrm{e}^{-\lambda P}\right)-\left(\varepsilon+D_{2}\right)\right],
$$

where all parameters are positive constants. The boundary equilibria are $E_{0}=\left(N^{0}, 0,0\right)$ and and

$$
\begin{gathered}
\exp \left[-\frac{\lambda D\left(N^{0}-\left(\left(\gamma+D_{1}\right) k /\left(a-\left(\gamma+D_{1}\right)\right)\right)\right)}{\gamma+D_{1}-\gamma_{1}}\right] \\
<\frac{\delta c-\left(\varepsilon+D_{2}\right)}{\delta c}
\end{gathered}
$$

where

$$
\begin{aligned}
& P^{*}=\frac{1}{\lambda} \ln \frac{\delta c}{\delta c-\left(\varepsilon+D_{2}\right)}, \\
& Z^{*}=\frac{\delta P^{*}}{\varepsilon+D_{2}}\left[a \frac{N^{*}}{k+N^{*}}-\left(\gamma+D_{1}\right)\right]
\end{aligned}
$$

and $N^{*}$ is the positive root of the equation

$$
D\left(N^{*}\right)^{2}+B N^{*}-C=0
$$

with

$$
\begin{aligned}
B=D\left(k-N^{0}\right)+ & {\left[\left(\gamma+D_{1}-\gamma_{1}\right)\right.} \\
+ & \left.\delta\left(a-\gamma-D_{1}\right)\left(1-\frac{\varepsilon_{1}}{\varepsilon+D_{2}}\right)\right] P^{*}, \\
C=k\left\{D N^{0}+\right. & {\left[\delta\left(\gamma+D_{1}\right)\left(1-\frac{\varepsilon_{1}}{\varepsilon+D_{2}}\right)\right.} \\
& \left.\left.-\left(\gamma+D_{1}-\gamma_{1}\right)\right] P^{*}\right\} .
\end{aligned}
$$

$$
E_{1}=\left(\frac{\left(\gamma+D_{1}\right) k}{a-\left(\gamma+D_{1}\right)}, \frac{D\left(N^{0}-\left(\overline{\left.\left(\gamma+D_{1}\right) k /\left(a-\left(\gamma+D_{1}\right)\right)\right)}\right)\right.}{\gamma+D_{1}-\gamma_{1}}, 0\right)
$$

provided

$$
a>\gamma+D_{1} \quad \text { and } \quad N^{0}>\frac{\left(\gamma+D_{1}\right) k}{a-\left(\gamma+D_{1}\right)}
$$

The positive steady state $E^{*}=\left(N^{*}, P^{*}, Z^{*}\right)$ exists if

$$
\delta c>\varepsilon+D_{2}
$$

With parameter values given as (see Wroblewski \& Richman, 1987)

$$
\begin{gathered}
a=1.0, \quad k=0.2, \quad \gamma=0.1, \quad \varepsilon=0.1, \quad \lambda=0.5, \\
D=0.1, \quad D_{1}=0.1, \quad D_{2}=0.1, \\
c=0.5, \quad \gamma_{1}=0.08, \quad \varepsilon_{1}=0.05, \quad \delta=0.7,
\end{gathered}
$$

numerical simulations show that $N_{c}^{0}=8.4$ is the bifurcation value. When $N^{0}=4.0$, all three 


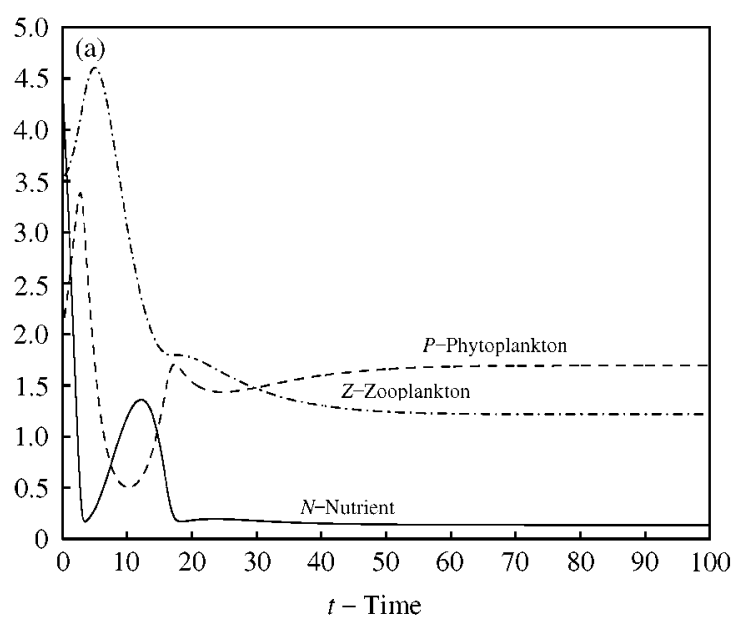

(b)
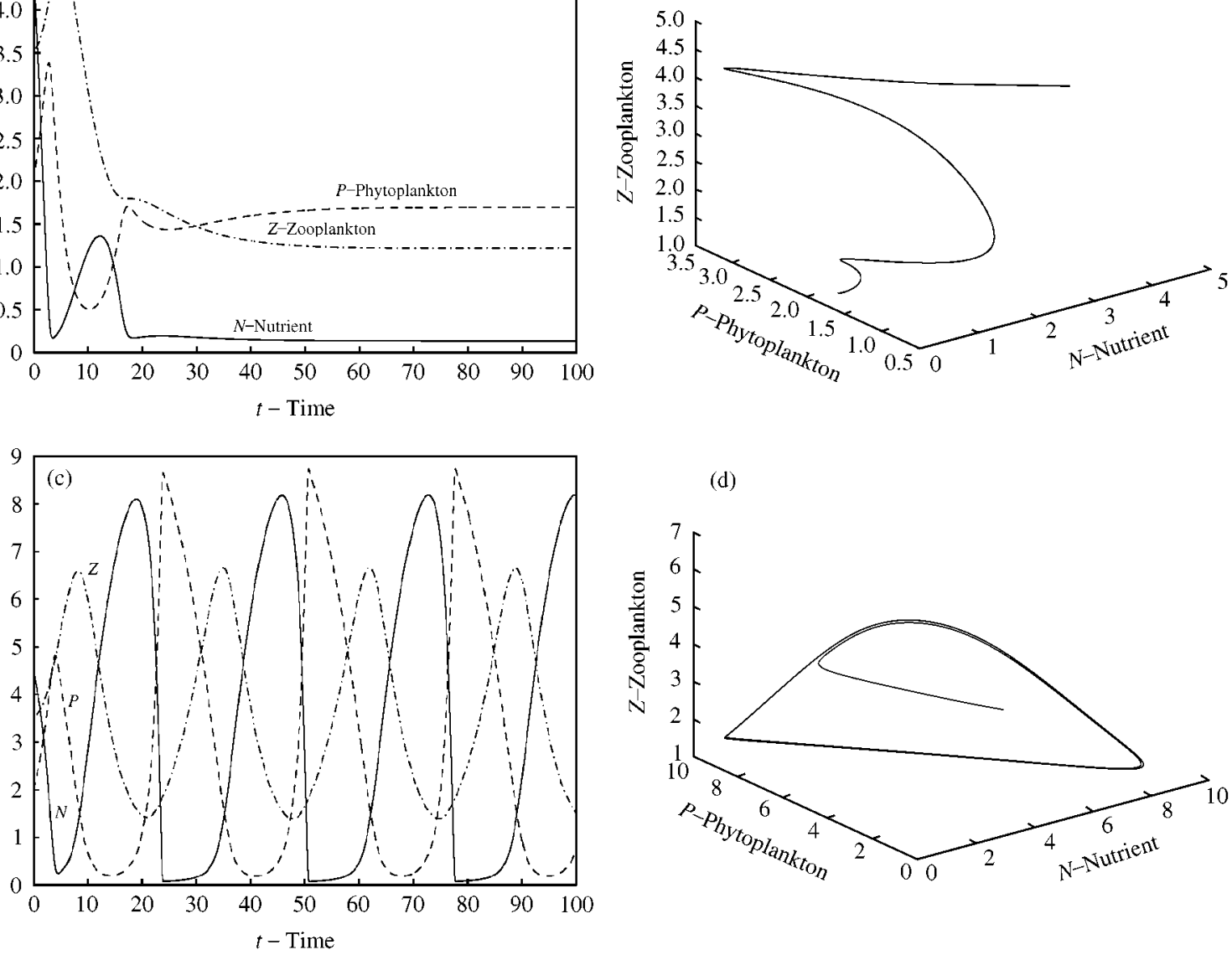

(d)

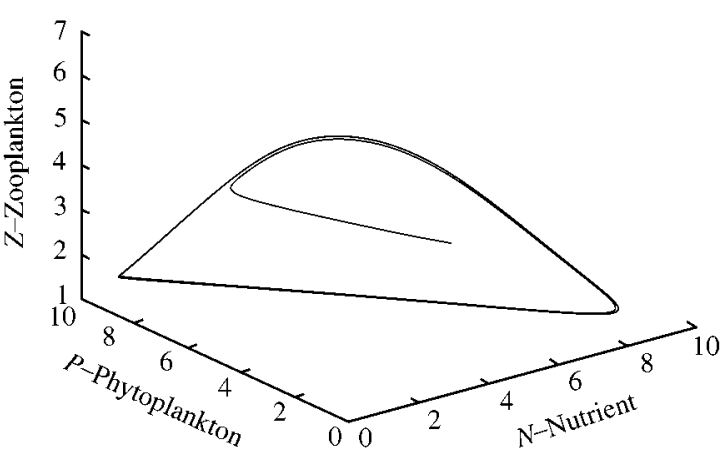

FIG. 1. Instantaneous nutrient recycling I.

components converge to the steady-state values [Fig. 1(a)] and the positive steady state is stable [Fig. 1(b)]. When $N^{0}=12.5>N_{c}^{0}$, all three components are oscillatory [Fig. 1(c)] and there is a periodic solution [Fig. 1(d)].

\section{Delayed Nutrient Recycling}

In this section, we consider the delay system (1). By Theorem 4.1 in Ruan \& Wolkowicz (1995) we know that if

$$
\begin{aligned}
& \int_{-\infty}^{0} \int_{-\infty}^{s} F(u) \mathrm{d} u \mathrm{~d} s<\infty, \\
& \int_{-\infty}^{0} \int_{-\infty}^{s} G(u) \mathrm{d} u \mathrm{~d} s<\infty,
\end{aligned}
$$

then all solutions of system (1) are bounded.

Notice that the boundary equilibrium $E_{0}=$ $\left(N^{0}, 0,0\right)$ always exists. As in Section 3 , if the

inequalities in eqn (10) hold, then the boundary equilibrium $E_{1}=\left(N_{1}, P_{1}, 0\right)$ exists, where $N_{1}$ and $P_{1}$ are the same as given in Section 3. Furthermore, if the inequalities in eqn (12) hold, then there is a positive equilibrium $E^{*}=\left(N^{*}, P^{*}, Z^{*}\right)$.

To discuss the local stability of the positive equilibrium $E^{*}$, we consider a specific case. Assume that $u(N)$ is the Michaelis-Menten function, $w(P)$ is the Ivlev function, and $F(s)$ and $G(s)$ re weak kernels, i.e. we consider the following model:

$$
\begin{aligned}
\frac{\mathrm{d} N}{\mathrm{~d} t}= & D\left(N^{0}-N\right)-a P \frac{N}{k+N} \\
& +(1-\delta) c Z\left(1-\mathrm{e}^{-\lambda P}\right) \\
& +\gamma_{1} \int_{-\infty}^{t} \alpha \mathrm{e}^{-\alpha(t-s)} P(s) \mathrm{d} s
\end{aligned}
$$




$$
+\varepsilon_{1} \int_{-\infty}^{t} \beta \mathrm{e}^{-\beta(t-s)} Z(s) \mathrm{d} s
$$

$$
\begin{aligned}
& \frac{\mathrm{d} P}{\mathrm{~d} t}=a P \frac{N}{k+N}-c Z\left(1-\mathrm{e}^{-\lambda P}\right)-\left(\gamma+D_{1}\right) P, \\
& \frac{\mathrm{d} Z}{\mathrm{~d} t}=Z\left[\delta c\left(1-\mathrm{e}^{-\lambda P}\right)-\left(\varepsilon+D_{2}\right)\right],
\end{aligned}
$$

where all parameters are positive numbers. If

$$
\begin{gathered}
a>\gamma+D_{1}, \\
\delta c>\varepsilon+D_{2}, \\
N^{0}>\frac{\left(\gamma+D_{1}\right) k}{a-\left(\gamma+D_{1}\right)}
\end{gathered}
$$
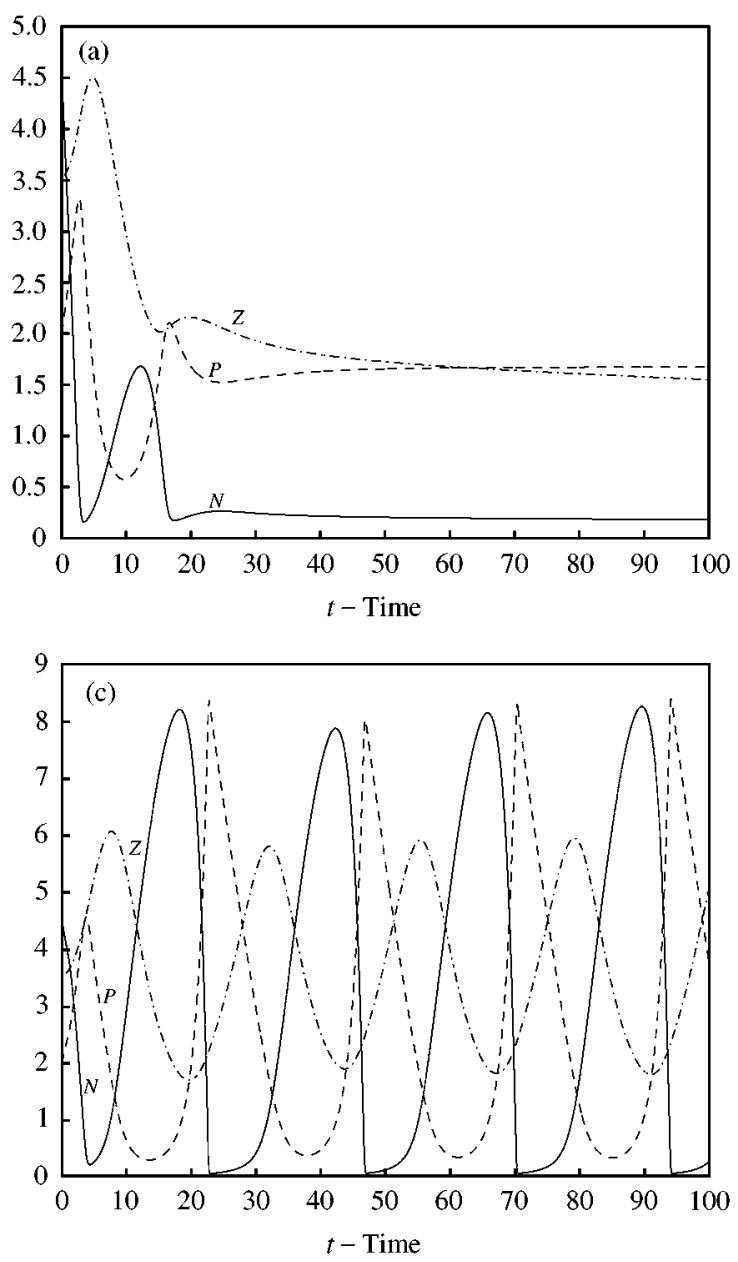

FIG. 2. Delayed nutrient recycling. and

$$
\begin{gathered}
\exp \left[-\frac{\lambda D\left(N^{0}-\left(\left(\gamma+D_{1}\right) k /\left(a-\left(\gamma+D_{1}\right)\right)\right)\right)}{\gamma+D_{1}-\gamma_{1}}\right] \\
<\frac{\delta c-\left(\varepsilon+D_{2}\right)}{\delta c}
\end{gathered}
$$

then by Theorem 4.5 in Ruan \& Wolkowicz (1995), system (24) is uniformly persistent and there is a positive equilibrium $E^{*}=\left(N^{*}, P^{*}, Z^{*}\right)$, which is the same equilibrium of system (20) given by eqns (21) and (22). By using the so-called "linear chain trick" technique (see MacDonald, 1978), the characteristic equation of the linearized system at $E^{*}$ can be written as

$$
y^{5}+b_{1} y^{4}+b_{2} y^{3}+b_{3} y^{2}+b_{4} y+b_{5}=0,
$$

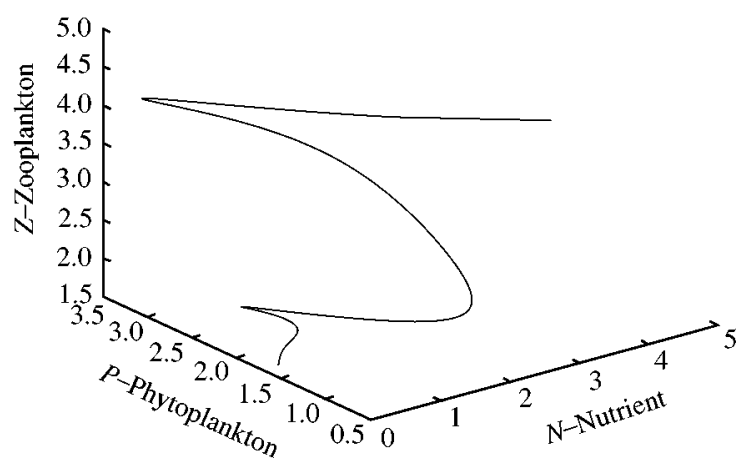

(d)

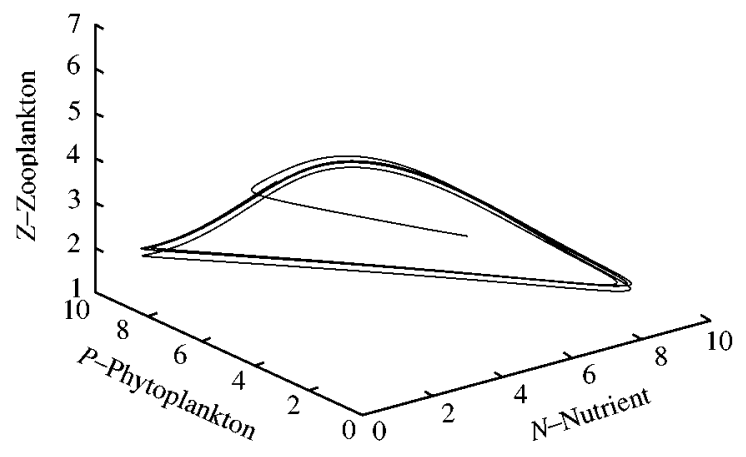


where

$$
\begin{aligned}
& b_{1}=\alpha+\beta+a_{1}, \\
& b_{2}=\alpha \beta+(\alpha+\beta) a_{1}+a_{2}, \\
& b_{3}=\alpha \beta a_{1}+(\alpha+\beta) a_{2}+a_{3}+\alpha \gamma_{1} m_{21}, \\
& b_{4}=\alpha \beta\left(\gamma_{1} m_{21}+a_{2}\right)+(\alpha+\beta) a_{3}+\beta \varepsilon_{1} m_{21} m_{32}, \\
& b_{5}=\alpha \beta\left(a_{3}+\gamma_{1} m_{21}+\varepsilon_{1} m_{21} m_{32}\right),
\end{aligned}
$$

with $a_{i}(i=1,2,3)$ and $m_{i j}(i, j=1,2,3)$ defined in eqns (13) and (11).

By using the Routh-Hurwicz criteria, we can determine the linear stability of the positive equilibrium $E^{*}$. Choosing $N^{0}$ as a bifurcation parameter and following a similar analysis as in Section 3 , we can show that $E^{*}$ could lose its stability when $E^{*}$ passes a critical value $N_{c}^{0}$ and a Hopf bifurcation could occur. Numerical simulations confirm the observation. With parameter values chosen as

$$
\alpha=0.02, \quad \beta=0.01
$$

and all other parameters given in Section 3, numerical simulations are depicted in Fig. 2(a)-(d). When $N^{0}=4.0$, all three components tend to the steady-state values and the equilibrium is stable [Fig. 2(a) and (b)]. When $N^{0}=12.5>N_{c}^{0}=8.4$, the three components coexist in an oscillatory mode [Fig. 2(c) and (d)].

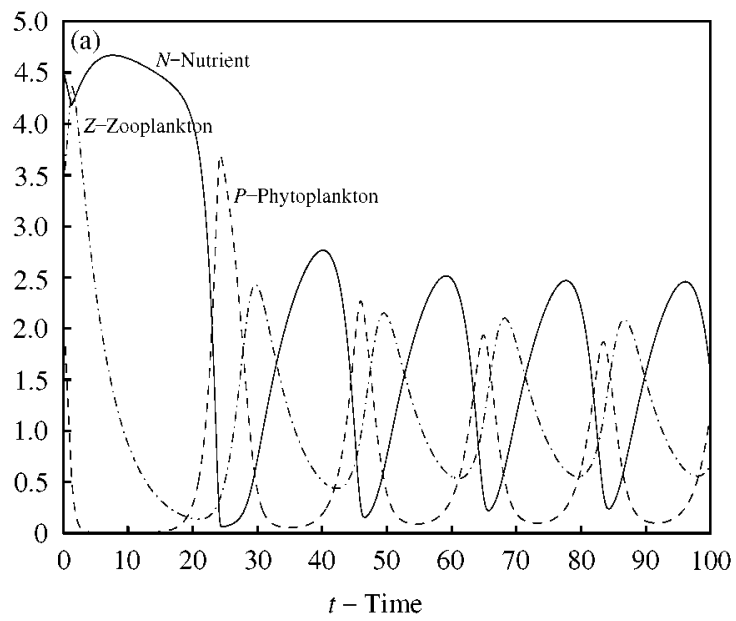

FIG. 3. Instantaneous nutrient recycling II.
Comparing Fig. 1(a) with 2(a), we can see that for the stable steady state, time delays induce more transient oscillations. In the case of periodic solutions [see Figs 1(c) and 2(c)], we can see that delays in the nutrient recycling make the oscillations more frequently.

\section{Discussion}

We have studied plankton-nutrient models with both instantaneous and delayed nutrient recycling. Local stability of the equilibria has been analysed. By choosing the nutrient input concentration, $N^{0}$, as a bifurcation parameter, we have shown that the positive equilibrium loses its stability when $N^{0}$ passes a critical value and a Hopf bifurcation occurs at the positive equilibrium. Recall that the uniform persistence criteria of the system are given by inequalities (24)-(28). Conditions (27) and (28) can be rewritten as

$$
\begin{aligned}
N^{0}> & \frac{\left(\gamma+D_{1}\right) k}{a-\left(\gamma+D_{1}\right)} \\
& \quad+\frac{\gamma+D_{1}-\gamma_{1}}{\lambda D} \ln \frac{\delta c}{\delta c-\left(\varepsilon+D_{2}\right)},
\end{aligned}
$$

which indicates that the models are nutrient controlled (see Hallam, 1978). Therefore, if the nutrient input concentration is sufficient, then the system is uniformly persistent. However, our analysis shows that if the input concentration surpasses a threshold value, the system becomes

(b)

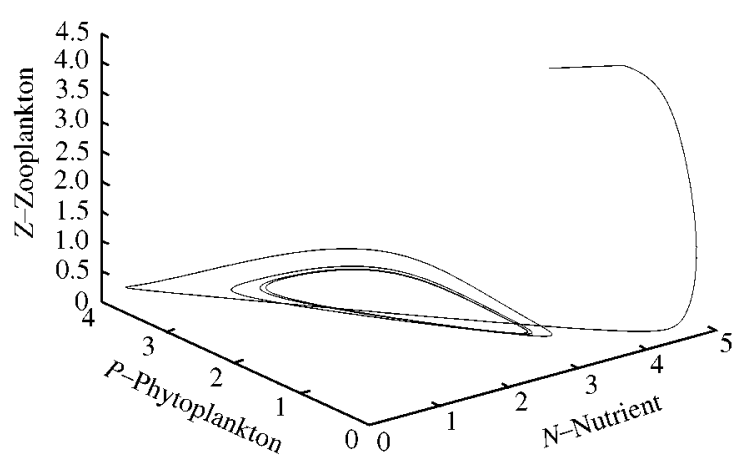


unstable and the plankton populations start to oscillate. This reminds one of the phenomenon of the "paradox of enrichment".

If the maximal zooplankton ingestion rate, $c$, is used as a bifurcation parameter, one can also observe similar bifurcations. With $c=1.25$ and all other parameters the same as in Section 3 for Fig. 1(a) and (b), numerical simulations are depicted in Fig. 3(a) and (b). This indicates that the codimension of these bifurcations might be greater than 1 and the plankton models could exhibit very interesting and complicated dynamics (see Edwards, 1997; Edwards \& Brindley, 1996; Popova et al., 1997; Scheffer, 1991, and the references therein).

\section{REFERENCES}

Beretta, E., Bischi, G. I. \& Solimano, F. (1990). Stability in chemostat equations with delayed nutrient recycling. J. Math. Biol. 28, 99-111.

Beretta, E., Fergola, P. \& Tenneriello, C. (1995). Chemostat equations for a predator-prey chain with delayed nutrient recycling. J. Biol. Systems 3, 483-494.

BERETTA, E. \& TAKEUCHI, Y. (1994a). Qualitative properties of chemostat equations with time delays: boundedness, local and global asymptotic stability. Differential Equations Dyn. Systems 2, 19-40.

BeretTA, E. \& TAKEUCHI, Y.(1994b). Qualitative properties of chemostat equations with time delays II. Differential Equations Dyn. Systems 2, 263-288.

BISCHI, G. I. (1992). Effects of time lags on transient characteristics of a nutrient cycling model. Math. Biosci. 109, 151-175.

Busenberg, S., Kumar, S. K., Austin, P. \& Wake, G. (1990). The dynamics of a model of a plankton-nutrient interaction. Bull. Math. Biol. 52, 677-696.

Cushing, J. M. (1977). Integrodifferential Equations and Delay Models in Population Dynamics. Heidelberg: SpringerVerlag.

DeAngelis, D. L., Bartell, S. M. \& Brenkert, A. L. (1989). Effects of nutrient recycling and food-chain length on resilience. Am. Nat. 134, 778-805.

EDWARDS, A. M. (1997). A rational dynamical-systems approach to plankton population modelling. Ph.D. Thesis, University of Leeds.

EDWARDS, A. M. \& BRINDLEY, J. (1996). Oscillatory behavior in a three-component plankton population model. Dyn. Stability Systems 11, 347-370.

Evans, G. T. \& PARSlOw, J. S. (1985). A model of annual plankton cycles. Biol. Oceanogr. 3, 327-347.

FASHAM, M. J. R. (1993). Modelling the marine biota. In: The Global Carbon Cycle (Heimann, M. ed), pp. 457-504. Berlin: Springer-Verlag.

Fasham, M. J. R., Ducklow, H. W. \& McKelvie, S. M. (1990). A nitrogen-based model of plankton dynamics in the oceanic mixed layer. J. Mar. Res. 48, 591-639.

Franks, P. J. S., Wroblewski, J. S. \& Flierl, G. R. (1986). Behavior of a simple plankton model with food-level acclimation by herbivores. Mar. Biol. 91, 121-129.
Freedman, H. I. \& XU, Y. (1993). Models of competition in the chemostat with instantaneous and delayed nutrient recycling. J. Math. Biol. 31, 513-527.

Hale, J. K. \& Somolinos, A. S. (1983). Competition for fluctuating nutrient. J. Math. Biol. 18, 255-280.

Hallam, G. (1978). Structural sensitivity of grazing formulation in nutrient controlled plankton models. J. Math. Biol. 5, 261-280.

HE, X. \& RUAN, S. (1998). Global stability in chemostat-type plankton models with delayed nutrient recycling. J. Math. Biol. 37, 253-271.

He, X., RuAn, S. \& XIA, H. (1998). Global stability in chemostat-type equations with distributed delays. SI AM J. Math. Anal. 29, 681-696.

Henderson, E. W. \& Steele, J. H. (1995). Comparing models and observations of shelf plankton. J. Plankton Res. 17, 1679-1692.

Holling, C. S. (1959). Some characteristics of simple type of predation and parasitism. Canad. Entomol. 91, 385-395.

IVLEV, V. S. (1961). Experimental Ecology of the Feeding of Fishes. New Haven: Yale University Press.

MacDonald, N. (1978). Time Lags in Biological Models. Heidelberg: Springer-Verlag.

Mayzaud, P. \& Poulet, S. A. (1978). The importance of the time factor in the response of zooplankton to varying concentrations of naturally occurring particulate matter. Limnol. Oceanogr. 23, 1144-1154.

Nisbet, R. M. \& GuRNEy, W. S. C. (1976). Model of material cycling in a closed ecosystem. Nature 264, 633-635.

Nisbet, R. M., Mckinstry, J. \& Gurney, W. S. C. (1983). A strategic model of material cycling in a closed ecosystem. Math. Biosci. 64, 99-113.

Popova, E. E., Fasham, M. J. R., Osipov, A. V. \& RyabCHENKO, V. A. (1997). Chaotic behaviour of an ocean ecosystem model under seasonal external forcing. J. Plankton Res. 19, 1495-1515.

Powell, T. \& Richerson, P. J. (1985). Temporal variation, spatial heterogeneity and competition for resource in plankton system: a theoretical model. Am. Nat. 125, 431-464.

RUAN, S. (1993). Persistence and coexistence in zooplankton-phytoplankton-nutrient models with instantaneous nutrient recycling. J. Math. Biol. 31, 633-654.

RUAN, S. (1995). The effect of delays on stability and persistence in plankton models. Nonlinear Anal. 24, 575-585.

RUAN, S. \& HE, X.-Z. (1998). Global stability in chemostattype competition models with nutrient recycling. SIAM J. Appl. Math. 58, 170-192.

RUAN, S. \& WOLKOWICZ, G. (1995). Uniform persistence in plankton models with delayed nutrient recycling. Canad. Appl. Math. Quart. 3, 219-235.

Sarmiento, J. L., Slater, R. D., Fasham, M. J. R., DuCKLOW, H. W., Toggweiler, J. R. \& Evans, G. T. (1993). A seasonal three-dimensional ecosystem model of nitrogen cycling in the North Atlantic euphotic zone. Global Biogeochem. Cycles 7, 417-450.

SCHEFFER, M. (1991). Should we expect strange attractors behind plankton dynamics and if so, should we bother? J. Plankton Res. 13, 1291-1305.

Steele, J. H. \& Henderson, E. W. (1981). A simple plankton model. Am. Nat. 117, 676-691.

Steele, J. H. \& Henderson, E. W. (1992). The role of predation in plankton models. J. Plankton Res. 14, 157-172. 
Svirezhev, Y. M. \& Logofet, D. O. (1983). Stability of Biological Communities. Moscow: MIR.

TruscotT, J. E. \& BRINDley, J. (1994). Equilibria, stability and excitability in a general class of plankton population models. Phil. Trans. Roy. Soc. London A 347, 703-718.

WhitTaker, R. H. (1975). Communities and Ecosystems. New York: Macmillan.
Wroblewski, J. S. \& Richman, J. G. (1987). The non-linear response of plankton to wind mixing events - implications for survival of larval northern anchovy. J. Plankton Res. 9, 103-123. Wroblewski, J. S., Sarmiento, J. L., \& Flierl, G. R. (1988). An ocean basin scale model of plankton dynamics in the North Atlantic 1. Solutions for the climatological oceanographic conditions in May. Global Biogeochem. Cycles 2, 199-218. 